\title{
Association between IL-6 and metabolic syndrome in schizophrenia patients treated with second-generation antipsychotics
}

This article was published in the following Dove Press journal:

Neuropsychiatric Disease and Treatment

\section{Xinyu Fang \\ Yewei Wang \\ Yan Chen \\ Juanjuan Ren \\ Chen Zhang}

Schizophrenia Program, Shanghai Mental Health Center, Shanghai Jiao Tong University School of Medicine, Shanghai, People's Republic of China
Correspondence: Chen Zhang Schizophrenia Program, Shanghai Mental Health Center, Shanghai Jiao Tong University School of Medicine, 600 Wan Ping Nan Road, Shanghai, People's Republic of China

Email zhangchen645@gmail.com
Objective: Second-generation antipsychotics (SGAs) have a high risk of causing metabolic syndrome (MetS). There is accumulating evidence supporting the fact that the activation of inflammatory pathway contributes to the development of MetS and further aggravates cognitive impairment. This study aimed to investigate the relationship between interleukin6 (IL-6), cognitive function, and MetS in schizophrenia patients treated with SGAs.

Methods: One hundred and seventy-four patients with schizophrenia using SGAs were divided into MetS and non-MetS group, based on the criteria of the National Cholesterol Education Program's Adult Treatment Panel III. Cognitive function was measured using the Repeatable Battery for the Assessment of Neuropsychological Status (RBANS). A total of 138 patients and 29 healthy controls were examined in the plasma IL-6 levels.

Results: The prevalence of MetS in schizophrenia patients treated with SGAs was $33 \%$ in this study. There were no significant differences in cognitive functions (both RBANS total score and subscale score) between MetS and non-Mets patients $(P>0.05)$. Patients with MetS had higher plasma levels of IL-6 compared to non-MetS patients $(P=0.019)$. However, such difference was only found in male patients (male: $P=0.012$; female: $P=0.513$ ). The partial correlational analysis further showed that IL-6 levels were notably negative related to the HDL levels in male schizophrenia patients after age, years of education, body mass index (BMI), age of onset, total disease course, and equal dose of olanzapine were controlled (male: $P=0.009$; female: $P=0.450$ ). In addition, the multiple regression analysis (stepwise model) performed in the male patient subgroup showed that IL-6 (beta $=-0.283, \mathrm{t}=-2.492$, $P=0.015$ ) was an independent contributor to the HDL levels. However, the IL-6 was not an independent contributor to the HDL levels in female patients.

Conclusion: Our findings provide evidence suggesting that the immune-inflammatory effect of IL-6 on SGAs-induced MetS may be in a gender manner.

Keywords: schizophrenia, interleukin-6, metabolic syndrome, second-generation antipsychotics

\section{Introduction}

Schizophrenia is a chronic and deliberating disorder, characterized by positive and negative symptoms and cognitive impairment. ${ }^{1}$ It affects approximately $1 \%$ of the world's population and increased social burden, thus, becoming a serious public health problem. ${ }^{2}$ Antipsychotic drug has been widely acknowledged as the first-line treatment for schizophrenia since first-generation antipsychotics (FGAs) were found to have advantages in improving positive symptoms in the 1950s. ${ }^{3}$ Compared to FGAs, second-generation antipsychotics (SGAs) lead to a lower risk 
of extrapyramidal symptoms and tardive dyskinesia. ${ }^{4}$ Therefore, it has been more frequently prescribed for schizophrenia patients in recent years. Unfortunately, SGAs are more notorious than FGAs for causing metabolic syndrome (MetS), which is an important risk factor for cardiovascular disorders. ${ }^{5}$ These metabolic adverse effects are often associated with non-compliance and medical problems. ${ }^{6}$ Moreover, the average life span of people with schizophrenia is shortened by 20 years compared to individuals without this disorder. ${ }^{7}$ One of the possible explanations for the differential mortality rate is the increased risk of metabolic adverse effects in schizophrenia patients. ${ }^{8}$ Therefore, identifying biomarkers for MetS in schizophrenia patients and to reduce its occurrence remains an important goal.

MetS is described as a cluster of obesity-related risk factors for chronic metabolic and cardiovascular diseases including abdominal obesity, hypertension, hyperglycemia, insulin resistance, increased triglycerides, and decreased high-density lipoprotein (HDL) cholesterol based on the National Cholesterol Education Program-Adult Treatment Panel III (NCEP-ATP III) criteria. ${ }^{9}$ The data from the Clinical Antipsychotic Trial of Intervention Effectiveness (CATIE) have shown that the prevalence of MetS for schizophrenia in the United States is $40.9 \%,{ }^{10}$ which is significantly higher compared to the general population. ${ }^{11}$ MetS and its components are important risk factors for developing cognitive impairment and dementia. ${ }^{12,13}$ Our previous preliminary findings indicated that schizophrenia patients with MetS had poorer cognitive functions than those without MetS, and immune-inflammation may play an important role in this regard. ${ }^{4}$ And previous studies have also supported the role of immune inflammation in MetS and cognitive impairment. ${ }^{14}$ However, not all studies revealed a significant relationship between MetS (and its components) and cognitive impairment in schizophrenia. For example, Bora and colleagues failed to find any association between MetS and cognition through a largest CATIE trial. ${ }^{15}$ Interestingly, hyperglycemia, an important component in MetS, was actually found to predict better verbal memory performance. ${ }^{16}$ Hence, it is not clear whether or not cognitive deficits in schizophrenia are specifically associated with MetS.

Ample literature has documented that immune deregulation may lead to the cluster of chronic metabolic disorders, ${ }^{17}$ and aberrant peripheral levels of immuneinflammatory markers also reported in schizophrenia patients used in SGAs. ${ }^{18}$ Thus, chronic low-grade inflammation may be involved in the pathogenesis of MetS in schizophrenia patients. Abdominal obesity is considered as a marker of dysfunctional adipose tissue, as well as an important manifestation of MetS. ${ }^{19}$ Interleukin-6 (IL-6), as the chief stimulator of the response in inflammation, it is reported that appropriately one-third of circulating IL-6 has been attributed to adipose tissue secretion, with macrophages being the major contributor. ${ }^{20}$ During the acute and chronic inflammatory states, the secretion of IL-6 from adipose tissue highly increased, which suggests a link between IL- 6 and obesity. ${ }^{20}$ And early researches also supported a possible relationship between IL-6 and MetS in patients with schizophrenia; ${ }^{21,22}$ however, there are conflicting results. ${ }^{18,23}$

Therefore, the aim of the present study was to evaluate and compare plasma levels of IL- 6 and cognitive functions between schizophrenia with and without MetS, and to investigate the relationship between MetS, especially its components, and IL-6 in schizophrenia patients. We hypothesized that patients with MetS would have higher plasma IL-6 levels than those without MetS, and IL-6 may be associated with specific metabolic indicators.

\section{Methods}

\section{Participants}

This is a cross-sectional study conducted in Shanghai Mental Health Center. A total of 174 (82 males and 92 females) inpatients with schizophrenia were included in our present study. All patients met the following criteria: (1) had been diagnosed with schizophrenia according to the Diagnostic and Statistical Manual of Mental Disorders, Fourth Edition (DSM-IV) with the diagnoses made by experienced psychiatrists; (2) age 18-50 years, Han Chinese; (3) had a junior high school education or above; (4) patients were receiving SGAs treatment only. They were excluded if they: (1) had other diagnosed psychiatric disorder besides schizophrenia or a lifetime substance abuse/dependence disorder; (2) uncontrolled major medical conditions, including any comorbid medical disease that could potentially influence the immune system before 1 month prior to study entry; (3) were pregnant or breastfeeding. We also recruited 29 (12 males and 17 females) healthy individuals for controls, who were screened by a specialized psychiatrist using the Structured Clinical Interview for DSM-IV-TR Axis I Disorders-Patient Edition. The dosage of antipsychotics was recalculated to become an equivalent dose of 
olanzapine according to defined daily dose (DDD) method developed by the World Health Organization (WHO). This study was reviewed and approved by the Institutional Review Boards of the Shanghai Mental Health Center, and all procedures were performed in strict accordance with the Declaration of Helsinki and other relevant national and international regulations. Moreover, the written informed consent was obtained from each participant prior to the performance of any procedures related to this study.

\section{Clinical assessment}

We collected the demographic and clinical data of subjects through self-made questionnaires, and basic demographic and clinical information of schizophrenia patients were obtained from medical records and case managers. The Positive and Negative Syndrome Scale (PANSS) was used for measuring the severity of psychotic symptoms exhibited by the schizophrenia patients. Two experienced psychiatrists had simultaneously attended a training session on using PANSS and repeated assessments for the PANSS total score maintained and inter-rater correlation coefficient greater than 0.8 .

\section{Cognitive tests}

The Repeatable Battery for the Assessment of Neuropsychological Status (RBANS) was applied to evaluate cognitive functioning in schizophrenia patients. ${ }^{24}$ The 12-item RBANS make up of a total score and 5 ageadjusted index scores, including immediate memory (list learning and story memory tasks), visuospatial/constructional (figure copy and line orientation tasks), language (picture naming and semantic fluency tasks), attention (digit span and coding tasks), and delayed memory (list recall, story recall, figure recall, and list recognition tasks). It has good validity and reliability in Chinese people ${ }^{25}$ and works well in cognitive evaluations of patients with schizophrenia. ${ }^{26,27}$

\section{Anthropometric and biochemical parameters}

MetS was diagnosed based on the presence of any three of the following criteria: (1) a waist circumference $\geq 90 \mathrm{~cm}$ in Chinese men and $\geq 80 \mathrm{~cm}$ in Chinese women; (2) triglyceride (TG) $\geq 1.7$ $\mathrm{mmol} / \mathrm{L}$; (3) HDL cholesterol $<1.0 \mathrm{mmol} / \mathrm{L}$ in men and $<1.3$ $\mathrm{mmol} / \mathrm{L}$ in women; (4) blood pressure $\geq 130 / 85 \mathrm{mmHg}$; or (5) fasting glucose $(\mathrm{GLU}) \geq 5.6 \mathrm{mmol} / \mathrm{L}$. The above criterion is based on the definition by the NCEP-ATP III and is also the best MetS criterion for a Chinese population. ${ }^{28}$

Following an overnight fast, serum samples from the patients were collected between 6:00 and 9:00 a.m. and stored at $-80^{\circ} \mathrm{C}$ until used for the assay. Serum fasting GLU, TG, and HDL levels were measured using an automatic Biochemical Analyzer (HITACHI 7170A, Hitachi, Ltd, Tokyo, Japan). Blood pressure including systolic blood pressure (SBP) and diastolic blood pressure (DBP) was also measured using an automated blood pressure machine before the blood collection. And waist circumference was measured between the lower rib margin and the iliac crest, after a normal expiratory breath as described in our previous article. ${ }^{29}$

\section{Plasma IL-6 measurements}

A total of $10 \mathrm{~mL}$ fasting blood samples was collected from 138 patients and 29 healthy controls between 06:00 and 09:00 a.m. The plasma was separated by centrifugation at $3000 \mathrm{r} / \mathrm{min}$ for $15 \mathrm{mins}$ at $4^{\circ} \mathrm{C}$ and stored at $-80^{\circ} \mathrm{C}$ until used for assay. Plasma levels of IL-6 were measured with the Quantibody Human Inflammation Array 1 (QAH-INF-1) from Ray Biotech (Norcross, GA) according to the manufacturer's instructions. ${ }^{30}$ And the limitation of detection was $1 \mathrm{pg} / \mathrm{mL}$ for IL-6 with this measurement.

\section{Statistical analysis}

Statistical analyses were performed using SPSS v. 23.0. After excluding a potential outlier of IL-6 ( $\geq$ Mean $\pm 3 \times \mathrm{SD}, 240.01 \mathrm{pg} / \mathrm{m}$ ), the Kolmogorov-Smirnov one-sample test was used to examine the normality of the biochemical parameters, and IL-6 levels in patients and normal controls $(P>0.05)$. We then used the independent Student's $t$-test or analysis of variance (ANOVA) for continuous variables and chi-square for categorical variables to compare the differences in demographic, clinical variables, and blood parameters between groups. And the analysis of covariance (ANCOVA) was performed to control the effects of confounding variables. In addition, the correlation between IL-6 and other variables was calculated by Pearson's correlation analysis, and a partial correlational analysis was used to explore the relationship between IL-6 and components of MetS, controlling for age, sex, BMI, age of onset, total course, and equal dose of olanzapine. Then, the multiple regression analysis (stepwise model) was used to identify significant predictive variables associated with the component of MetS in schizophrenia patients. Finally, we further performed sex 
stratification to explore whether sex difference exists in the relationship between IL-6 and MetS or its components. And the Bonferroni-adjusted significance tests were used for multiple comparisons. All tests were two-tailed and with statistical significance as $P<0.05$. And we used the G*Power 3.1.9.2 program (https://www.softpedia.com/get/ Science-CAD/G-Power.shtml) to run a power calculation and determine the effect size.

\section{Results}

The demographic characteristics for schizophrenia patients and healthy controls are summarized in Table 1 . There were no significant differences in terms of age, sex, years of education, marital status, and body mass index (BMI) between the two groups. Among 138 patients measured the plasma IL-6 levels, only 137 were included in the final analysis due to one potential outlier was excluded. Our results showed that patients had a markedly higher IL-6 levels compared to healthy controls $(9.14 \pm 2.52$ vs 7.98 $\pm 2.96 \mathrm{pg} / \mathrm{mL}, \mathrm{F}(1,165)=4.801, P=0.030)$. After age, sex, years of education were controlled as covariance, this difference still remained $(\mathrm{F}=4.303, P=0.040)$ (Figure 1A). And the power calculation showed that the statistical power reached $74 \%$.

Among the 174 schizophrenia patients receiving SGAs treatment, MetS was found in 57 (33\%) patients. The demographic, clinical features, and biochemical parameters of the MetS and non-MetS groups are presented in Table 2. Our results showed that MetS patients had a higher BMI, waistline, SBP, DBP, serum fasting GLU, TG levels (all $P<0.001)$, and a longer total disease course $(P=0.001)$ compared to non-MetS patients. However, patients with MetS had a lower level of HDL than non-MetS patients $(P<0.001)$. To compare the plasma IL-6 levels between

Table I Characteristics of patients with schizophrenia and healthy controls

\begin{tabular}{|l|l|l|l|l|}
\hline & $\begin{array}{l}\text { Patients } \\
(\mathbf{N}=174)\end{array}$ & $\begin{array}{l}\text { Controls } \\
\mathbf{( N = 2 9 )}\end{array}$ & Statistics & $\boldsymbol{P}$ \\
\hline Age (year) & $35.83 \pm 9.52$ & $33.24 \pm 5.99$ & $\mathrm{t}=1.956$ & 0.056 \\
Sex (male/female) & $82 / 92$ & $12 / 17$ & $\mathrm{X}^{2}=0.330$ & 0.688 \\
Education (year) & $13.06 \pm 8.84$ & $14.59 \pm 3.34$ & $\mathrm{t}=0.919$ & 0.359 \\
BMI (kg/m $\left.{ }^{2}\right)$ & $24.07 \pm 3.78$ & $23.19 \pm 3.30$ & $\mathrm{t}=1.186$ & 0.237 \\
Marital status & $117 / 57$ & $16 / 13$ & $\mathrm{X}^{2}=1.603$ & 0.291 \\
(single/married) & & & & \\
IL-6 (pg/mL) & $9.14 \pm 2.52$ & $7.98 \pm 2.96$ & $\mathrm{~F}=4.80 \mathrm{I}$ & 0.030 \\
\hline
\end{tabular}

Note: Data presented as mean \pm SD or $\mathrm{N}$.

Abbreviations: BMI, body mass index; IL-6, interleukin 6 . patients with and without MetS (44 vs 93), we found that MetS patients had significantly higher IL-6 levels than nonMetS patients $(9.90 \pm 2.62$ vs $8.78 \pm 2.40 \mathrm{pg} / \mathrm{mL}, \mathrm{F}=6.036$, $P=0.015$ ), and these differences remained even after we controlled for age, sex, years of education, BMI, age of onset, total disease course, and equal dose of olanzapine $(\mathrm{F}=5.609, P=0.019)$ (Figure 1B). Moreover, the significant difference also withstood the Bonferroni correction $(P=0.019<0.05 / 2)$. However, there were no significant differences in age, sex, years of education, BMI, marital status, episode (first or recurrence), family history of mental illness, antipsychotics (monotherapy or drug combination), and equal dose of olanzapine between those two groups $(P>0.05)$. There were also no significant differences in the severity of psychotic symptoms (PANSS total or subscale score) and cognitive functions (RBANS total or subscale score) between the MetS and non-MetS groups $(P>0.05)$.

For the correlation analysis conducted in the schizophrenia patients, we found that IL-6 was not associated with demographic data and clinical variables such as age, years of education, BMI, age of onset, total disease course, and equal dose of olanzapine $(P>0.05)$. And we also found that IL-6 was not correlated with the severity of psychotic symptoms (PANSS total or subscale score) as well as cognitive functions (RBANS total or subscale score) $(P>0.05)$. For components of MetS, our results showed a negative correlation between IL-6 and HDL levels ( $\mathrm{r}=-0.185, P=0.030)$, but not $\mathrm{BP}$, waistline, fasting GLU, and TG levels $(P>0.05)$ (Table S1). The results of the partial correlational analysis further showed that plasma IL-6 levels were notably related to the HDL levels in schizophrenia patients after controlling for age, sex, years of education, BMI, age of onset, total disease course, and equal dose of olanzapine as covariates $(\mathrm{r}=-0.190, P=0.030)$ (Figure 2A). Further multiple regression analysis (stepwise model) performed showed that BMI (beta $=-0.188, \mathrm{t}=-2.402$, $P=0.018$ ), sex (beta $=0.340, \mathrm{t}=4.350, P=0.001$ ) and IL-6 (beta $=-0.180, \mathrm{t}=-2.318, P=0.022$ ) were independent contributors to the HDL levels in schizophrenia patients.

Since we found sex was significantly associated with HDL levels in schizophrenia patients, further analyses were performed separately for the women and men with schizophrenia to assess sex differences in IL-6 associated with HDL. For male patients, our results showed that IL-6 levels were significantly higher in MetS patients compared to nonMetS patients ( $\mathrm{F}=6.709, P=0.012)$ after age, years of education, BMI, age of onset, total disease course, and equal dose of olanzapine were controlled (see Figure 1C and D). And this significant difference also withstood the Bonferroni 

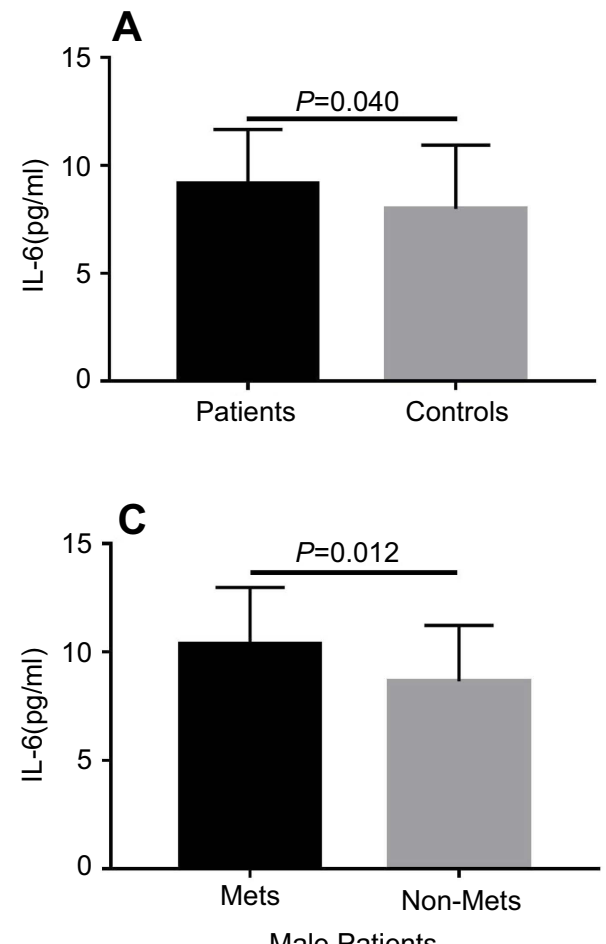
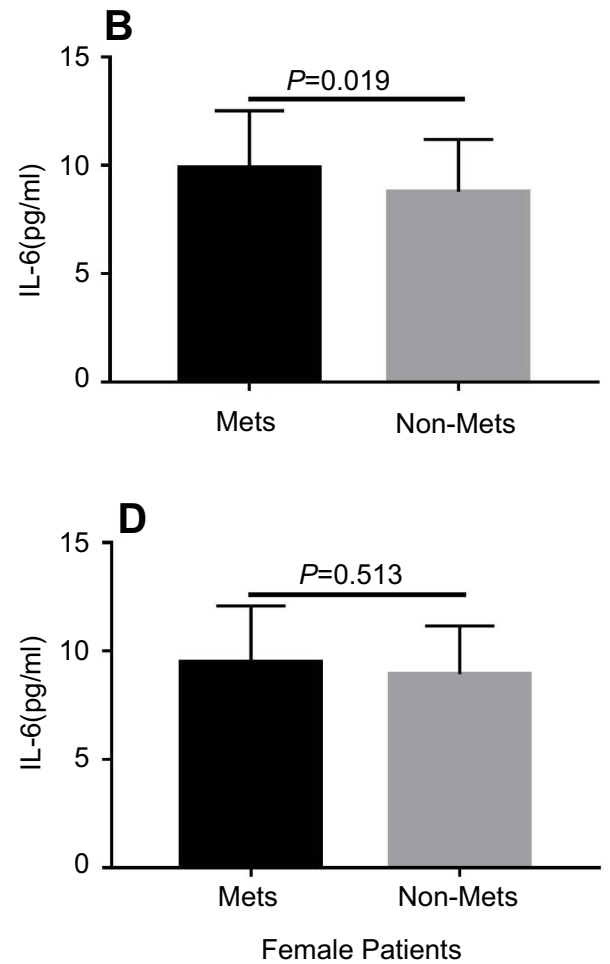

Figure I Comparison of plasma IL-6 levels between different groups. Each bar represents the mean level of IL-6. Error bars represent the standard deviation. (A) Plasma level of IL-6 in schizophrenia patients and healthy controls $(9.14 \pm 2.52$ vs $7.98 \pm 2.96 \mathrm{pg} / \mathrm{mL}, \mathrm{F}=4.303, P=0.040)$; (B) Plasma level of IL-6 in MetS and non-MetS groups ( 9.90 \pm 2.62 vs $8.78 \pm 2.40 \mathrm{pg} / \mathrm{mL}, F=5.609, P=0.019) ;(C)$ Plasma levels of IL-6 in male MetS patients and non-MetS patients (I0.35 $\pm 2.63 \mathrm{vs} 8.66 \pm 2.57 \mathrm{pg} / \mathrm{mL}, \mathrm{F}=6.709, P=0.012) ;(\mathbf{D})$ Plasma levels of IL-6 in female MetS patients and non-MetS patients $(9.48 \pm 2.60$ vs $8.93 \pm 2.23 \mathrm{pg} / \mathrm{mL}, \mathrm{F}=0.403, P=0.5 \mathrm{I} 3)$. The $P$-values for intergroup comparison of IL-6 were adjusted for age, sex, years of education, BMI, age of onset, total disease course, and equal dose of olanzapine were controlled as covariates.

Abbreviations: BMI, body mass index; IL-6, interleukin 6; MetS, metabolic syndrome.

correction $(P=0.012<0.05 / 2)$. There was a negative correlation between IL- 6 and HDL levels in male patients after age, years of education, BMI, age of onset, total disease course, and equal dose of olanzapine were controlled $(\mathrm{r}=-0.321$, $P=0.009$ ) (Figure 2B). For female patients, after age, years of education, BMI, age of onset, total disease course, and equal dose of olanzapine were controlled, no significant difference in IL-6 levels between MetS patients and nonMetS patients $(\mathrm{F}=0.403, P=0.513)$ was found. And there was also no significant association between IL- 6 and HDL levels in female patients (female: $\mathrm{r}=-0.099, P=0.450$ ). Finally, the multiple regression analysis (stepwise model) performed in the male patient subgroup showed that BMI (beta $=-0.236$, $\mathrm{t}=-2.079, P=0.041$ ) and IL-6 (beta $=-0.283, \mathrm{t}=-2.492$, $P=0.015)$ were independent contributors to the HDL levels. However, the IL-6 was not an independent contributor to the HDL levels in female patients.

\section{Discussion}

In the present study, the overall prevalence of MetS in the 174 patients with SGAs was 33\%, which is similar to the rate $(32.5 \%)$ reported in a recent meta-analysis, ${ }^{31}$ while lower than that $(41.8 \%)$ reported by our previous study with the monotherapy with clozapine $(43.2 \%)^{29}$ or olanzapine $(44 \%) .{ }^{4}$ Although all SGAs lead to high risk of MetS, with clozapine and olanzapine are the most obesogenic. ${ }^{32}$ Therefore, the lower rate of MetS may be due to the fact that our patients also used other SGAs in addition to olanzapine and clozapine.

The results of our study have shown that MetS patients had higher plasma IL-6 levels compared to those without MetS. IL-6 is an important pro-inflammatory cytokine and plays a vital role in the immune response. ${ }^{33}$ Therefore, the elevated levels of IL-6 indicated a high inflammatory state in schizophrenia patients with MetS. Inflammation has 
Table 2 Demographics and clinical features between MetS and non-MetS patients

\begin{tabular}{|c|c|c|c|c|}
\hline & MetS $(\mathrm{N}=57)$ & Non-MetS $(\mathbf{N}=\mid 17)$ & $\mathbf{F} / \mathbf{X}^{2}$ & $P$ \\
\hline Age (year) & $37.79 \pm 9.45$ & $34.88 \pm 9.44$ & 6.036 & 0.015 \\
\hline Sex (male/female) & $23 / 34$ & $59 / 58$ & 1.562 & 0.258 \\
\hline Education (year) & $12.40 \pm 2.87$ & $13.38 \pm 10.59$ & 0.463 & 0.497 \\
\hline BMI $\left(\mathrm{kg} / \mathrm{m}^{2}\right)$ & $26.01 \pm 3.46$ & $23.13 \pm 3.57$ & 25.452 & $<0.001$ \\
\hline Marital status (single/married) & $36 / 21$ & $81 / 36$ & 0.642 & 0.492 \\
\hline Age of onset (year) & $23.30 \pm 6.85$ & $23.77 \pm 6.65$ & 0.188 & 0.665 \\
\hline Total course (month) & $|80.5| \pm 10 \mid .76$ & $|30.07 \pm 9| .63$ & 10.80 & 0.001 \\
\hline Episode (first/recurrence) & $3 / 54$ & $6 / 111$ & 0.001 & 1.000 \\
\hline Family history (no/yes) & $47 / 10$ & $81 / 36$ & 3.447 & 0.069 \\
\hline Antipsychotics (single/combination) & $32 / 25$ & $67 / 50$ & 0.020 & 1.000 \\
\hline Equal dose of olanzapine $(\mathrm{mg})$ & $16.12 \pm 6.54$ & $|8.12 \pm 7.0|$ & 3.269 & 0.072 \\
\hline Waist circumference $(\mathrm{cm})$ & $92.37 \pm 9.87$ & $83.84 \pm 8.50$ & 34.657 & $<0.001$ \\
\hline Fasting GLU (mmol/L) & $5.98 \pm 1.79$ & $4.90 \pm 0.79$ & 30.679 & $<0.001$ \\
\hline Fasting TG (mmol/L) & $2.23 \pm 1.05$ & $1.24 \pm 0.94$ & 39.131 & $<0.001$ \\
\hline Fasting HDL (mmol/L) & $1.03 \pm 0.20$ & $1.24 \pm 0.33$ & 20.289 & $<0.001$ \\
\hline $\mathrm{SBP}(\mathrm{mm} \mathrm{Hg})$ & $121.86 \pm 10.23$ & || $4.11 \pm|0.7|$ & 20.640 & $<0.001$ \\
\hline $\mathrm{DBP}(\mathrm{mm} \mathrm{Hg})$ & $79.1 I \pm 8.27$ & $73.66 \pm 8.35$ & 16.424 & $<0.001$ \\
\hline \multicolumn{5}{|l|}{ PANSS } \\
\hline Positive symptom & $13.47 \pm 4.96$ & $13.00 \pm 4.43$ & 0.405 & 0.525 \\
\hline Negative symptom & $16.67 \pm 6.64$ & $16.88 \pm 5.94$ & 0.046 & 0.831 \\
\hline General psychopathology & $30.44 \pm 5.71$ & $30.60 \pm 6.62$ & 0.024 & 0.876 \\
\hline Total score & $60.88 \pm 12.92$ & $60.44 \pm 12.78$ & 0.044 & 0.835 \\
\hline \multicolumn{5}{|l|}{ RBANS } \\
\hline Immediate memory & $62.84 \pm 17.21$ & $61.69 \pm|5.5|$ & 0.196 & 0.659 \\
\hline Visuospatial skill & $68.42 \pm 10.91$ & $67.41 \pm 9.47$ & 0.394 & 0.531 \\
\hline Language & $71.28 \pm 18.04$ & $71.02 \pm 16.52$ & 0.009 & 0.924 \\
\hline Attention & $86.60 \pm 16.76$ & $87.49 \pm 16.49$ & 0.111 & 0.740 \\
\hline Delayed memory & $58.63 \pm|8.7|$ & $59.27 \pm 17.44$ & 0.049 & 0.824 \\
\hline Total score & $62.75 \pm 13.93$ & $62.50 \pm 11.92$ & 0.015 & 0.902 \\
\hline
\end{tabular}

Notes: Data presented mean \pm SD or $\mathrm{N}$.

Abbreviations: MetS, metabolic syndrome; BMI, body mass index; GLU, glucose; TG, triacylglyceride; HDL, high-density lipoprotein; SBP, systolic pressure; DBP, diastolic pressure; PANSS, Positive and Negative Syndrome Scale; RBANS, Repeatable Battery for the Assessment of Neuropsychological Status.

been proposed to be of major pathophysiological importance for the development of MetS, and IL-6 have repeatedly been associated with MetS in general population. ${ }^{34,35}$ Previous preclinical study also suggested that schizophrenia-relevant metabolic abnormalities could be primed by immune activation, and pro-inflammatory IL-6 is an important indicator involved in those processes. ${ }^{36}$ In addition, early literature demonstrated that inflammation is one of the most important mechanisms proposed for the development of clozapine-related MetS, and evidence derived from that clozapine promote the release of IL- 6 in insulinresponsive cells and obesity-associated cell types. ${ }^{22}$ To further figure out which component among the five metabolic indicators was most associated with IL-6, we performed a correlation analysis and only found a significantly negative correlation between IL-6 and HDL before and after age, sex, years of education, BMI, age of onset, total disease course, and equal dose of olanzapine were all controlled. Our further multiple regression analysis also showed that IL-6 was an independent contributor to the HDL levels in schizophrenia. The negative correlation between IL- 6 and HDL has also been reported by other studies. ${ }^{37,38}$ Therefore, our results suggest that the effect of IL-6 on MetS may be related to the specific regulation of HDL.

Schizophrenia displays sex difference in many aspects, such as age of onset, ${ }^{39}$ suicide rate, ${ }^{40}$ cognitive function, ${ }^{41}$ inflammatory characteristic, ${ }^{42}$ and MetS. ${ }^{43}$ Interestingly, our results showed that the elevated IL-6 levels were only found in male patients with MetS, and there was a 
A

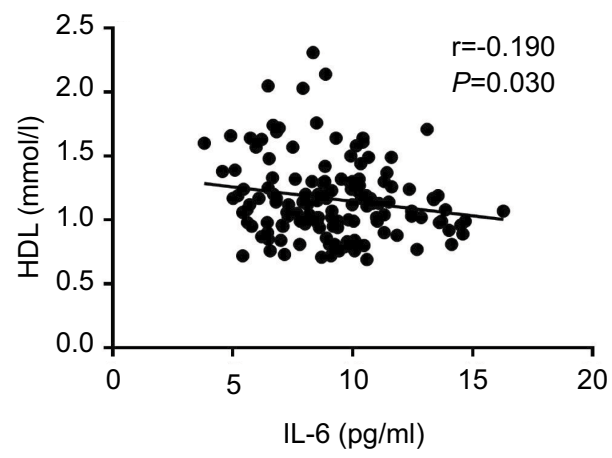

B

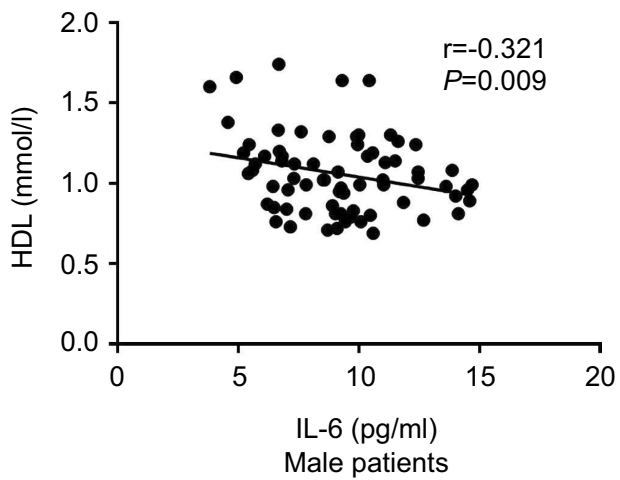

Figure 2 Correlation between plasma IL-6 and HDL levels. (A) Correlation between plasma IL-6 and HDL levels in patients group ( $r=-0.190, P=0.030)$; (B) Correlation between plasma IL-6 and HDL levels in male patients group $(r=-0.321, P=0.009)$.

Abbreviations: IL-6, interleukin 6; HDL, high-density lipoprotein.

significant correlation between IL-6 levels and HDL in males, but not in females. To the best of our knowledge, sex difference in the immune-inflammatory characteristics of MetS in schizophrenia has received little systematic study. However, ample evidence supports sex characteristics of MetS in patients with schizophrenia. ${ }^{43-45}$ And some studies found that male schizophrenia patients may be associated with higher metabolic risk, but lower antioxidant enzyme activities than females. ${ }^{4-46}$ This difference may come from the protection of sex hormone against the oxidative stress and immune inflammation in females. ${ }^{47}$ Hence, male patients presumably are more liable to consequences of the immune activation and the release of IL6 , and to further leads to the occurrence of MetS in male patients. However, since the sample size of the male and female groups decreased after sex stratification, our conclusions need to be verified in a larger sample.

Since evidence has shown that the MetS and its components may lead to poorer cognitive functioning in general population, ${ }^{12}$ a number of studies have been done to investigate the effects of the components of MetS on cognitive function in schizophrenia patients recently, but the results are contradictory. ${ }^{4,15,48}$ In the present study, we did not detect any association between MetS or its component and cognitive functioning in schizophrenia. Other studies including the CATIE study also did not find the association between MetS and cognitive domains. ${ }^{15}$ As such, the relationship between the MetS and cognitive function is inconsistent. It should be mentioned that there are significant methodological differences across studies when investigating the effects of MetS components on cognition in schizophrenia, especially the fact that almost all studies are cross-sectional, which preclude any direct causal relationship between MetS and cognition. Thus, the conflict results have to be confirmed with longitudinal studies.

Several limitations of the present study should be mentioned here. First, the main limitation is obviously the crosssectional design, which could not demonstrate direct causal relationship between IL-6 and MetS in schizophrenia patients. Second, our sample size is small, especially after sex stratification; thus, the sex difference in the relationship between IL-6 and MetS should be viewed as preliminary results. Third, although the patients we included were all used SGAs, those drugs may exhibit different effects on metabolism, which may potentially confound the results obtained in the present study. However, due to the large variety of SGAs and our limited sample size, we were unable to conduct stratified analysis. Ultimately, future longitudinal study with larger study samples and further stratification of different drugs may provide a more adequate understanding of the role of immune-inflammatory in schizophrenia patients with MetS.

In conclusion, our study found that schizophrenia patients with MetS had elevated plasma levels of IL-6 compared to patients without Mets, and IL-6 was negatively correlated to HDL levels in the patients. However, the relationship between IL-6 and MetS, especially HDL, was only found in male patients. Our results suggest that sex is one of the important factors to consider when exploring the relationship between immune indicators and metabolic parameters in schizophrenia. And further 
longitudinal studies with a larger sample size are warranted to replicate these findings.

\section{Acknowledgments}

We are deeply grateful to all of the patients and healthy controls participating in this study as well as to the psychiatrists for their help in the recruitment and diagnosis of schizophrenic patients. This work was supported by the National Key Research and Development Program of China (2018YFC1314302), the National Natural Science Foundation of China (81771450, 81471358), the Shanghai Science and Technology Commission Foundation (14411969000), the Shanghai Municipal Education Commission-Gaofeng Clinical Medicine Grant Support (20152530), the Shanghai Municipal Commission of Health and Family Planning Foundation (201540029) and the Shanghai Municipal Commission of Health and Family Planning, Key Developing Disciplines (2015ZB0405).

\section{Disclosure}

The authors report no conflicts of interest in this work.

\section{References}

1. Kuperberg G, Heckers S. Schizophrenia and cognitive function. Curr Opin Neurobiol. 2000;10:205-210.

2. Zhang W, Amos TB, Gutkin SW, Lodowski N, Giegerich E, Joshi K. A systematic literature review of the clinical and health economic burden of schizophrenia in privately insured patients in the United States. Clinicoecon Outcomes Res. 2018;10:309-320. doi:10.2147/ CEOR.S156308

3. Zhang C, Li Z, Shao Y, et al. Association study of tryptophan hydroxylase-2 gene in schizophrenia and its clinical features in Chinese Han population. J Mol Neurosci. 2011;43:406-411. doi:10.1007/s12031010-9458-2

4. Zhang C, Fang X, Yao P, et al. Metabolic adverse effects of olanzapine on cognitive dysfunction: a possible relationship between BDNF and TNF-alpha. Psychoneuroendocrinology. 2017;81:138-143. doi:10.1016/j.psyneuen.2017.04.014

5. Hirsch L, Yang J, Bresee L, Jette N, Patten S, Pringsheim T. Secondgeneration antipsychotics and metabolic side effects: a systematic review of population-based studies. Drug Saf. 2017;40:771-781. doi:10.1007/s40264-017-0543-0

6. Jeon SW, Kim YK. Unresolved issues for utilization of atypical antipsychotics in schizophrenia: antipsychotic polypharmacy and metabolic syndrome. Int J Mol Sci. 2017;18(10): 2174.

7. Laursen TM, Nordentoft M, Mortensen PB. Excess early mortality in schizophrenia. Annu Rev Clin Psychol. 2014;10:425-448. doi:10.1146/ annurev-clinpsy-032813-153657

8. Bushe C, Holt R. Prevalence of diabetes and impaired glucose tolerance in patients with schizophrenia. Br J Psychiatry Suppl. 2004;47: S67-S71.

9. Lone S, Lone K, Khan S, Pampori RA. Pampori, Assessment of metabolic syndrome in Kashmiri population with type 2 diabetes employing the standard criteria's given by WHO, NCEPATP III and IDF. J Epidemiol Glob Health. 2017;7:235-239. doi:10.1016/j. jegh.2017.07.004
10. Meyer JM. Strategies for the long-term treatment of schizophrenia: real-world lessons from the CATIE trial. $J$ Clin Psychiatry. 2007;68:28-33.

11. Bora E, Akdede BB, Alptekin K. The relationship between cognitive impairment in schizophrenia and metabolic syndrome: a systematic review and meta-analysis. Psychol Med. 2017;47:1030-1040. doi: $10.1017 /$ S0033291716003366

12. Qiu C, Fratiglioni L. A major role for cardiovascular burden in agerelated cognitive decline. Nat Rev Cardiol. 2015;12:267-277. doi:10.1038/nrcardio.2014.223

13. Kaffashian S, Dugravot A, Elbaz A, et al. Predicting cognitive decline: a dementia risk score vs. the Framingham vascular risk scores. Neurology. 2013;80:1300-1306.

14. MacKenzie NE, Kowalchuk C, Agarwal SM, et al. Antipsychotics, metabolic adverse effects, and cognitive function in schizophrenia. Front Psychiatry. 2018;9:622. doi:10.3389/fpsyt.2018.00622

15. Meyer JM, Nasrallah HA, McEvoy JP, et al. The Clinical Antipsychotic Trials Of Intervention Effectiveness (CATIE) schizophrenia trial: clinical comparison of subgroups with and without the metabolic syndrome. Schizophr Res. 2005;80(1):9-18. doi:10.1016/j. schres.2005.07.015

16. Goughari AS, Mazhari S, Pourrahimi AM, Sadeghi MM, Nakhaee N. Associations between components of metabolic syndrome and cognition in patients with schizophrenia. J Psychiatr Pract. 2015;21 (3):190-197. doi:10.1097/PRA.0000000000000065

17. Hotamisligil GS. Inflammation and metabolic disorders. Nature. 2006;444:860-867. doi:10.1038/nature05485

18. Beumer W, Drexhage RC, De Wit H, Versnel MA, Drexhage HA, Cohen D. Increased level of serum cytokines, chemokines and adipokines in patients with schizophrenia is associated with disease and metabolic syndrome. Psychoneuroendocrinology. 2012;37:19011911. doi:10.1016/j.psyneuen.2012.04.001

19. Madeshiya AK, Singh S, Dwivedi S, Konwar R, Natu SM, Ghatak A. Association of IL-10 gene $(-1082 \mathrm{~A}>\mathrm{G},-819 \mathrm{C}>\mathrm{T}$ and $-592 \mathrm{C}>\mathrm{A})$ polymorphism and its serum level with metabolic syndrome of north Indian subjects. $J$ Genet. 2017;96:53-64.

20. Neelamekam S, Nurjono M, Lee J. Regulation of interleukin-6 and leptin in schizophrenia patients: a preliminary analysis. Clin Psychopharmacol Neurosci. 2014;12:209-214. doi:10.9758/ cpn.2014.12.3.209

21. Klemettila JP, Kampman O, Seppala N, et al. Cytokine and adipokine alterations in patients with schizophrenia treated with clozapine. Psychiatry Res. 2014;218:277-283. doi:10.1016/j.psychres.2014.04.049

22. Contreras-Shannon V, Heart DL, Paredes RM, et al. Clozapineinduced mitochondria alterations and inflammation in brain and insulin-responsive cells. PLoS One. 2013;8:e59012. doi:10.1371/journal. pone. 0059012

23. Sirota P, Hadi E, Djaldetti M, Bessler H. Difference in inflammatory cytokine production by mononuclear cells from obese and non-obese schizophrenic patients. Acta Psychiatr Scand. 2015;132:301-305. doi:10.1111/acps. 12396

24. Randolph C, Tierney MC, Mohr E, Chase TN. The Repeatable Battery for the Assessment of Neuropsychological Status (RBANS): preliminary clinical validity. J Clin Exp Neuropsychol. 1998;20:310319. doi:10.1076/jcen.20.3.310.823

25. Cheng Y, Wu W, Wang J, Feng W, Wu X, Li C. Reliability and validity of the repeatable battery for the assessment of neuropsychological status in community-dwelling elderly. Arch Med Sci. 2011;7:850-857. doi:10.5114/aoms.2011.25561

26. Cai J, Zhu Y, Zhang W, Wang Y, Zhang C. Comprehensive family therapy: an effective approach for cognitive rehabilitation in schizophrenia. Neuropsychiatr Dis Treat. 2015;11:1247-1253. doi:10.2147/NDT. S 83569

27. Fang X, Chen Y, Wang Y, Zhang C. Identification of risk factors for suicidal ideation in patients with schizophrenia. Psychiatry Res. 2018;271:195-199. doi:10.1016/j.psychres.2018.11.051 
28. Zhou H, Guo ZR, Yu LG, et al. Evidence on the applicability of the ATPIII, IDF and CDS metabolic syndrome diagnostic criteria to identify CVD and T2DM in the Chinese population from a 6.3-year cohort study in mid-eastern China. Diabetes Res Clin Pract. 2010;90:319-325. doi:10.1016/j.diabres.2010.09.001

29. Zhang Y, Chen M, Chen J, et al. Metabolic syndrome in patients taking clozapine: prevalence and influence of catechol-O-methyltransferase genotype. Psychopharmacology (Berl). 2014;231:22112218. doi:10.1007/s00213-013-3410-4

30. Zheng J, Shi Y, Xiong L, et al. The expression of IL-6, TNF-alpha, and MCP-1 in respiratory viral infection in acute exacerbations of chronic obstructive pulmonary disease. $J$ Immunol Res. 2017;2017:8539294. doi:10.1155/2017/5974574

31. Mitchell AJ, Vancampfort D, Sweers K, van Winkel R, Yu W, De Hert M. Prevalence of metabolic syndrome and metabolic abnormalities in schizophrenia and related disorders - a systematic review and metaanalysis. Schizophr Bull. 2013;39:306-318. doi:10.1093/schbul/sbr148

32. Vancampfort D, Stubbs B, Mitchell AJ, et al. Risk of metabolic syndrome and its components in people with schizophrenia and related psychotic disorders, bipolar disorder and major depressive disorder: a systematic review and meta-analysis. World Psychiatry. 2015;14:339-347. doi:10.1002/wps.20252

33. Zhang C, Wu Z, Zhao G, Wang F, Fang Y. Identification of IL6 as a susceptibility gene for major depressive disorder. Sci Rep. 2016;6:31264. doi:10.1038/srep31264

34. Van Guilder GP, Hoetzer GL, Greiner JJ, Stauffer BL, DeSouza CA. Influence of metabolic syndrome on biomarkers of oxidative stress and inflammation in obese adults. Obesity (Silver Spring). 2016;14:2127-2131. doi:10.1038/oby.2006.248

35. Ingelsson $\mathrm{E}$, Hulthe $\mathrm{J}$, Lind L. Inflammatory markers in relation to insulin resistance and the metabolic syndrome. Eur J Clin Invest. 2008;38:502-509. doi:10.1111/j.1365-2362.2008.01962.x

36. Pacheco-Lopez G, Giovanoli S, Langhans W, Meyer U. Priming of metabolic dysfunctions by prenatal immune activation in mice: relevance to schizophrenia. Schizophr Bull. 2013;39:319-329. doi: $10.1093 / \mathrm{schbul} / \mathrm{sbr} 178$

37. Bian AL, Hu HY, Rong YD, Wang J, Wang J-X, Zhou X-Z. A study on relationship between elderly sarcopenia and inflammatory factors IL-6 and TNF-alpha. Eur J Med Res. 2017;22:25. doi:10.1186/ s40001-017-0266-9

38. Diaz-Ruiz M, Martinez-Triguero ML, Lopez-Ruiz A, et al. Metabolic disorders and inflammation are associated with familial combined hyperlipemia. Clin Chim Acta. 2019;490:194-199.
39. Venkatesh BK, Thirthalli J, Naveen MN, et al. Sex difference in age of onset of schizophrenia: findings from a community-based study in India. World Psychiatry. 2018;7:173-176. doi:10.1002/j.20515545.2008.tb00191.x

40. Ran MS, Mao WJ, Chan CL, et al. Gender differences in outcomes in people with schizophrenia in rural China: 14-year follow-up study. $\mathrm{Br}$ J Psychiatry. 2015;206:283-288. doi:10.1192/bjp.bp.113.139733

41. Zhang XY, Chen DC, Tan YL, et al. Gender difference in association of cognition with BDNF in chronic schizophrenia. Psychoneuroendocrinology. 2014;48:136-146. doi:10.1016/j. psyneuen.2014.06.004

42. Zhang XY, Zhou DF, Qi LY, et al. Superoxide dismutase and cytokines in chronic patients with schizophrenia: association with psychopathology and response to antipsychotics. Psychopharmacology (Berl). 2009;204:177-184. doi:10.1007/s00213-008-1447-6

43. Zhang Y, Chen M, Wu Z, et al. Association study of Val66Met polymorphism in brain-derived neurotrophic factor gene with clozapine-induced metabolic syndrome: preliminary results. PLoS One. 2013;8:e72652. doi:10.1371/journal.pone.0072652

44. Chen S, Broqueres-You D, Yang G, et al. Male sex may be associated with higher metabolic risk in first-episode schizophrenia patients: a preliminary study. Asian J Psychiatr. 2016;21:25-30. doi:10.1016/j. ajp.2015.12.001

45. Kraemer S, Minarzyk A, Forst T, Kopf D, Hundemer H-P. Prevalence of metabolic syndrome in patients with schizophrenia, and metabolic changes after 3 months of treatment with antipsychotics - results from a German observational study. BMC Psychiatry. 2011;11:173. doi:10.1186/1471-244X-11-173

46. Pepe H, Balci SS, Revan S, Akalin PP, Kurtoğlu F. Comparison of oxidative stress and antioxidant capacity before and after running exercises in both sexes. Gend Med. 2009;6:587-595. doi:10.1016/j. genm.2009.10.001

47. Harish G, Venkateshappa C, Mahadevan A, Pruthi N, Srinivas Bharath MM, Shankar SK. Glutathione metabolism is modulated by postmortem interval, gender difference and agonal state in postmortem human brains. Neurochem Int. 2011;59:1029-1042. doi:10.1016/ j.neuint.2011.08.024

48. Lindenmayer JP, Khan A, Kaushik S, et al. Relationship between metabolic syndrome and cognition in patients with schizophrenia. Schizophr Res. 2012;142:171-176. doi:10.1016/j.schres.2012. 09.019 


\section{Supplementary materials}

Table SI Correlations between demographic, clinical characteristics, metabolic components, and IL-6 levels in schizophrenia patients

\begin{tabular}{|c|c|c|}
\hline \multirow[t]{2}{*}{ Variable } & \multicolumn{2}{|c|}{ IL-6 levels } \\
\hline & $\mathbf{r}$ & $\mathbf{P}$ \\
\hline Age & 0.063 & 0.423 \\
\hline BMI & 0.017 & 0.827 \\
\hline Education & 0.030 & 0.698 \\
\hline Age of onset & 0.054 & 0.532 \\
\hline Total course & 0.089 & 0.300 \\
\hline Equal dose of olanzapine & -0.061 & $0.48 I$ \\
\hline PANSS total score & -0.146 & 0.089 \\
\hline Positive symptom & -0.019 & 0.822 \\
\hline Negative symptom & -0.105 & 0.221 \\
\hline General psychopathology & -0.149 & 0.083 \\
\hline RBANS total score & -0.059 & 0.493 \\
\hline Immediate memory & -0.054 & 0.531 \\
\hline Visuospatial skill & 0.048 & 0.575 \\
\hline Language & -0.062 & 0.474 \\
\hline Attention & -0.076 & 0.380 \\
\hline Delayed memory & -0.065 & 0.451 \\
\hline Waist circumference & -0.008 & 0.923 \\
\hline Fasting GLU & -0.006 & 0.947 \\
\hline Fasting TG & 0.133 & 0.122 \\
\hline Fasting HDL & -0.185 & 0.030 \\
\hline SBP & 0.094 & 0.274 \\
\hline DBP & 0.124 & 0.148 \\
\hline
\end{tabular}

Abbreviations: IL-6, interleukin 6; BMI, body mass index; GLU, glucose; TG, triacylglyceride; HDL, high-density lipoprotein; SBP, systolic pressure; DBP, diastolic pressure; PANSS, Positive and Negative Syndrome Scale; RBANS, Repeatable Battery for the Assessment of Neuropsychological Status.

\section{Publish your work in this journal}

Neuropsychiatric Disease and Treatment is an international, peerreviewed journal of clinical therapeutics and pharmacology focusing on concise rapid reporting of clinical or pre-clinical studies on a range of neuropsychiatric and neurological disorders. This journal is indexed on PubMed Central, the 'PsycINFO' database and CAS, and is the official journal of The International Neuropsychiatric Association (INA). The manuscript management system is completely online and includes a very quick and fair peer-review system, which is all easy to use. Visit http://www.dovepress.com/testimonials.php to read real quotes from published authors. 